\title{
Estudo anatômico das artérias perfurantes do músculo gastrocnêmio medial*
}

\author{
Anatomical study of perforator arteries \\ of the medial gastrocnemius muscle
}

\author{
Diogo Mesouita Rebouças ${ }^{1}$, LuCiano LuCindo da Silva ${ }^{1}$, Frederico Rodrigues Cunha ${ }^{1}$, \\ Fabiano InÁcio de Souza ${ }^{1}$, Frederico BarRa de Moraes ${ }^{1}$, Francisco Albino Rebouças Júnior ${ }^{2}$, \\ MÁRIO YOSHIHIDE KUWAE ${ }^{3}$, VALNEY LUÍS DA ROCHA ${ }^{4}$
}

\section{RESUMO}

Objetivos: Os autores apresentam os resultados da dissecção do feixe vascular do músculo gastrocnêmio medial (MGM) e de suas artérias perfurantes, com o objetivo de contribuir anatomicamente para seu estudo. Métodos: Foram dissecadas 13 peças englobando o joelho e perna (oito à direita e cinco à esquerda), de 13 cadáveres adultos (oito masculinos e cinco femininos), todos frescos. Foi realizado estudo anatômico e radiológico detalha-

* Trabalho realizado no Departamento de Ortopedia e Traumatologia do Hospital das Clínicas da Universidade Federal de Goiás UFG - Goiânia (GO), Brasil.

1. Médico Ortopedista do Hospital das Clínicas da Universidade Federal de Goiás - UFG - Goiânia (GO), Brasil.

2. Estagiário do Departamento de Ortopedia e Traumatologia do Hospital das Clínicas da Universidade Federal de Goiás - UFG Goiânia (GO), Brasil.

3. Chefe do Serviço de Cirurgia da Mão e Microcirurgia do Hospital das Clínicas da Universidade Federal de Goiás - UFG - Goiânia (GO), Brasil.

4. Chefe do Departamento de Ortopedia e Traumatologia do Hospital das Clínicas da Universidade Federal de Goiás - UFG - Goiânia (GO), Brasil.

Endereço para correspondência: Dr. Frederico Barra de Moraes, Departamento de Ortopedia e Traumatologia e Cirurgia Plástica do HC-FMUFG, Primeira Avenida, s/ nº, 3o andar, Setor Universitário

- 74605-085 - Goiânia, GO. Tel.: (62) 3269-8334.

E-mail: frederico_barra@yahoo.com.br

Recebido em 22/2/08. Aprovado para publicação em 8/4/08.

Copyright RBO2008 do avaliando o padrão de distribuição, trajeto, número e comprimento da irrigação do MGM e de suas artérias perfurantes. Resultado: Foi observado que a artéria sural medial (ASM) divide-se em dois troncos intramusculares principais (medial e lateral) e que em $90 \%$ dos casos as perfurantes provêm do tronco lateral. Em $\mathbf{3 8 , 5 \%}$ dos casos, foi identificada uma perfurante, ao passo que duas perfurantes são observadas em $\mathbf{4 6 , 1 \%}$ dos casos. Todas as artérias perfurantes estavam entre $5,7 \mathrm{~cm}$ e $14,0 \mathrm{~cm}$ da prega poplítea e entre $16,5 \mathrm{~cm}$ e $34,0 \mathrm{~cm}$ do maléolo medial. Conclusão: A irrigação do MGM pela ASM e suas artérias perfurantes faz-se adequada para sua utilização como retalho miocutâneo no membro inferior.

Descritores - Músculo esquelético/anatomia e histologia; Músculo esquelético/irrigação sanguínea; Dissecação; Cadáver; Retalhos cirúrgicos

\section{ABSTRACT}

Objectives: The authors present the results of dissecting the vascular bundle of the medial gastrocnemius muscle (MGM) and its perforating arteries with the purpose of giving their contribution to anatomical studies. Methods: 13 pieces of knee and leg (eight right and five left) of 13 fresh adult cadavers (eight male and five female) to evaluate the distribution, path, number and length of irrigation of the MGM and its perforating arteries. Results: They observed that the medial sural artery (ASM) is divided 
into two main intramuscular branches (medial and lateral), and in $90 \%$ of the cases, the perforating arteries come from the lateral branch. In $38.5 \%$ of the cases, they identified one perforating artery, and two perforating arteries were seen in $46.1 \%$ of the cases. All of the perforating arteries were at a distance of $14.0 \mathrm{~cm}$ from the popliteal fold and between $16.5 \mathrm{~cm}$ and $34.0 \mathrm{~cm}$ from the medial malleolus. Conclusion: $M G M$ irrigation by ASM and its perforating arteries is adequate for the use of the muscle as a myocutaneous flap of in the lower limb.

Keywords - Muscle, skeletal/anatomy \& histology; Muscle, skeletal/blood supply; Dissection; Cadaver; Surgical flaps

\section{INTRODUÇÃO}

As lesões do sistema músculo-esquelético com perda de substância são situações de difícil manejo terapêutico, sendo um desafio para a cirurgia reconstrutiva. $\mathrm{O}$ músculo gastrocnêmio medial (MGM) foi inicialmente utilizado como retalho muscular ou miocutâneo para as coberturas no membro inferior próximas ao joelho ${ }^{(1-6)}$.

$\mathrm{O}$ aprimoramento das técnicas cirúrgicas tem aumentado as opções de tratamento, com resultados cada vez melhores. Os retalhos dos ramos arteriais perfurantes musculocutâneos são cada vez mais utilizados. Em 1967, Fujino ${ }^{(7)}$ documentou a contribuição das perfurantes para a nutrição do interstício local, à semelhança da contribuição das artérias axiais. Em 1988, Koshima et $a l^{(8)}$ foram pioneiros na utilização de retalhos baseados em artérias perfurantes formados exclusivamente por pele e tecido celular subcutâneo. Porém, um verdadeiro retalho de perfurante requer, segundo Wei et $a l^{(9)}$, dissecção intramuscular para o seu uso prático.

Em 2001, Hallock ${ }^{(10)}$ descreveu as bases anatômicas do retalho baseado nas perfurantes do MGM, seguido pelos trabalhos de Cavadas em 2001 ${ }^{(11)}$ e Thione et al em 2004 ${ }^{(12)}$ de dissecção anatômica e injeção de resina nos vasos. Em nosso trabalho, a visualização da anatomia vascular do MGM e de suas artérias perfurantes se deu por dissecção e injeção de contraste radiopaco nos vasos e estudo das imagens radiológicas.
O objetivo deste trabalho é contribuir para o estudo anatômico da irrigação do MGM e avaliar a distribuição de seus vasos perfurantes, através do estudo radiológico contrastado dessa vascularização.

\section{MÉTODOS}

Foram realizadas dissecções anatômicas de 13 retalhos miocutâneos do MGM em 13 peças, englobando o joelho e perna (oito à direita e cinco à esquerda), de 13 cadáveres adultos (oito masculinos e cinco femininos), todos frescos, no Necrotério do Hospital das Clínicas da Universidade Federal de Goiás.

As dissecções do MGM foram realizadas com instrumental apropriado para cirurgia de mão e microcirurgia, segundo o que foi descrito por Moraes et $a l^{(13)}$.

As artérias perfurantes foram identificadas através de divulsão entre a fáscia e o ventre medial do músculo gastrocnêmio, desde sua borda anterior até sua divisão com o ventre lateral. A seguir, foi medida a distância das mesmas até a prega poplítea e maléolo medial.

A dissecção vascular intramuscular foi substituída pelo estudo radiográfico, com injeção de contraste radiológico (diatrizoatos de meglumina e de sódio) na artéria sural medial (ASM). As avaliações radiográficas foram realizadas em equipamento de mamografia que, a partir da densidade tecidual de cada peça, emitia quantidades adequadas de radiação, gerando excelentes imagens da ASM, seus principais ramos e perfurantes. A injeção de contraste e a avaliação pelo equipamento de mamografia foram possíveis em 10 das 13 peças anatômicas dissecadas (figuras 1, 2 e 3 ).

\section{RESULTADOS}

Foram estudados cadáveres com faixa etária média de 45,5 anos (idade variando entre 32 e 71 anos), sendo $61,5 \%$ do sexo masculino. O lado direito foi dissecado em $61,5 \%$ e o esquerdo, em $38,5 \%$.

A quantidade de ramos perfurantes variou de nenhum (em um caso) a três (também em um caso), com média de 1,5 (tabelas 1 e 2).

O estudo radiográfico da vascularização do MGM demonstrou dois troncos principais intramusculares (ramos medial e lateral), que se originam geralmente na porção onde a ASM penetra o músculo (hilo). Em 
90\% dos casos, foi observado que as perfurantes originaram-se do tronco intramuscular lateral (figura 4).

As perfurantes emergiram na fáscia muscular a uma distância média da prega poplítea de $10,4 \mathrm{~cm}$, varian-

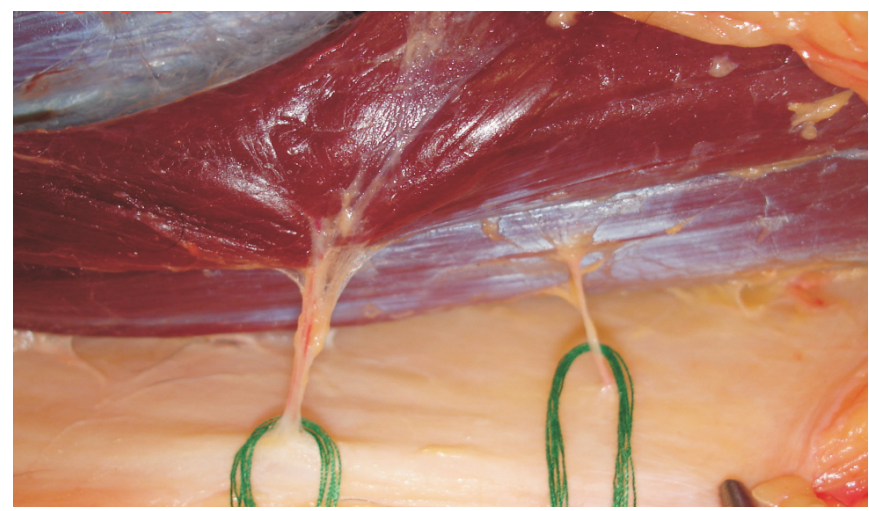

Figura 1 - Identificação anatômica dos ramos perfurantes

TABELA 1

Número de perfurantes e de troncos intermusculares

\begin{tabular}{rrllcc}
\hline $\begin{array}{c}\text { Caso } \\
\text { anos }\end{array}$ & $\begin{array}{c}\text { Sdade/ } \\
\text { anoxo }\end{array}$ & Lado & $\begin{array}{c}\text { Número de } \\
\text { perfurantes }\end{array}$ & $\begin{array}{c}\text { Número } \\
\text { de troncos } \\
\text { intramusculares }\end{array}$ \\
\hline 1 & 47 & masculino direito & 1 & não estudado \\
2 & 32 & feminino & direito & - & não estudado \\
3 & 35 & masculino direito & 1 & 2 \\
4 & 35 & masculino esquerdo & 2 & não estudado \\
5 & 35 & feminino & esquerdo & 2 & 1 \\
6 & 35 & feminino & direito & 2 & 2 \\
7 & 59 & feminino & direito & 2 & 2 \\
8 & 59 & feminino & esquerdo & 2 & 2 \\
9 & 40 & masculino direito & 1 & 2 \\
10 & 40 & masculino & esquerdo & 3 & 1 \\
11 & 52 & masculino direito & 1 & 1 \\
12 & 52 & masculino & esquerdo & 1 & 2 \\
13 & 71 & masculino direito & 2 & 2 \\
\hline
\end{tabular}

TABELA 2

Distribuições de perfurantes por peça

\begin{tabular}{ccc}
\hline $\begin{array}{c}\text { Número de } \\
\text { perfurantes por peça }\end{array}$ & $\begin{array}{c}\text { Número } \\
\text { de casos }\end{array}$ & $\begin{array}{c}\text { Percentual } \\
\text { de casos }\end{array}$ \\
\hline 0 & 1 & 7,7 \\
1 & 5 & 38,5 \\
2 & 6 & 46,1 \\
3 & 1 & 7,7 \\
\hline
\end{tabular}

do de 5,7 a $16,7 \mathrm{~cm}$, enquanto a distância ao maléolo medial variou de 16,5 a $36,0 \mathrm{~cm}$, com média de $28,4 \mathrm{~cm}$ (tabela 3).

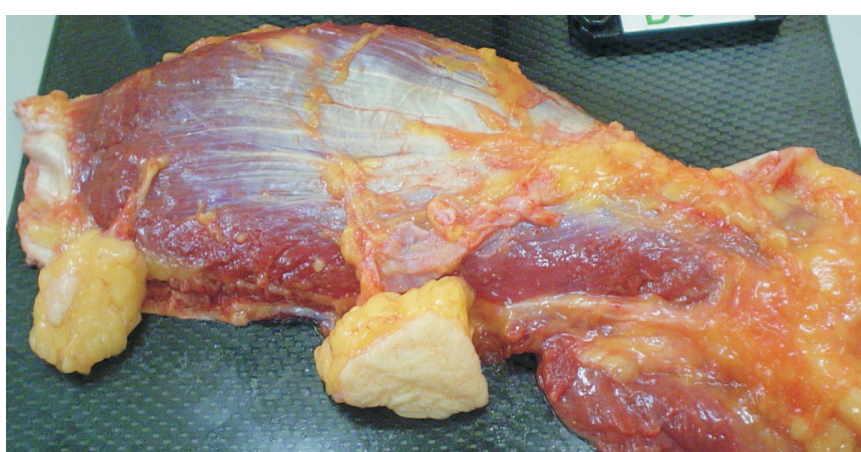

Figura 2 - Peça anatômica posicionada no equipamento de mamografia

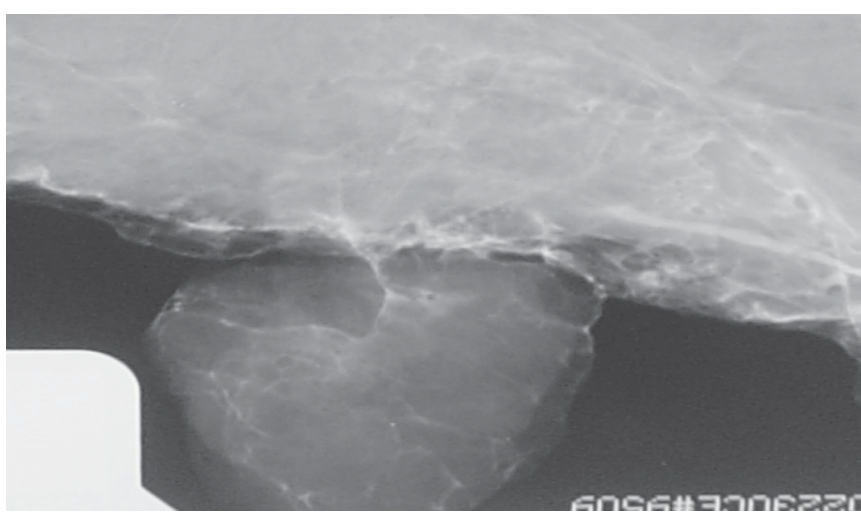

Figura 3 - Presença do contraste radiológico no seguimento fasciocutâneo

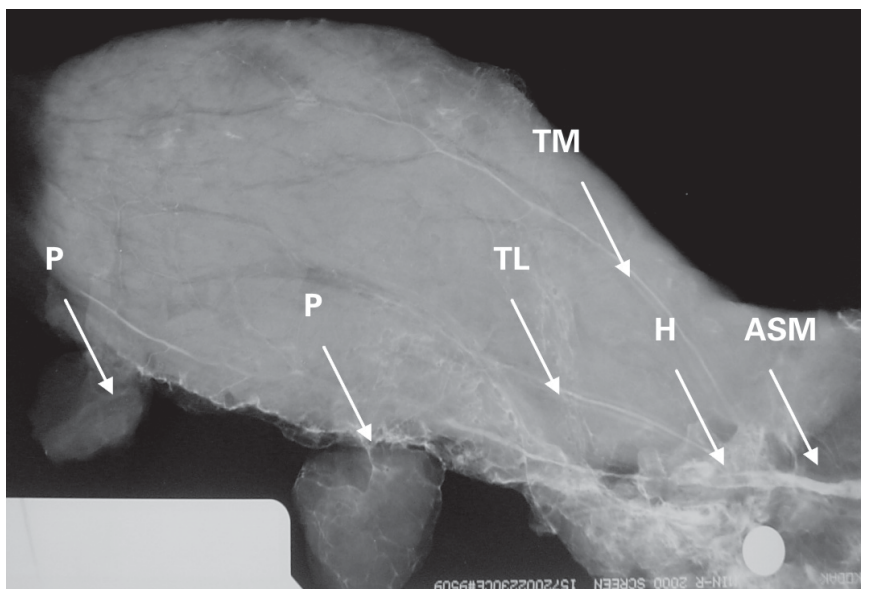

Figura 4 - Mamografia da peça mostrando dois troncos intramusculares e as perfurantes $(\mathrm{TL}=$ tronco lateral, $\mathbf{T M}=$ tronco medial, $\mathbf{H}=$ hilo, $\mathbf{A S M}=$ artéria sural medial, $\mathbf{P}=$ perfurantes) 
TABELA 3

Distância emergencial de perfurantes

\begin{tabular}{clcc}
\hline Caso & Perfurante & $\begin{array}{c}\text { Distância da } \\
\text { prega poplítea }\end{array}$ & $\begin{array}{c}\text { Distância do } \\
\text { maléolo medial }\end{array}$ \\
\hline 1 & Única & $7,5 \mathrm{~cm}$ & $29,0 \mathrm{~cm}$ \\
2 & Nenhuma & - & - \\
3 & Única & $7,5 \mathrm{~cm}$ & $33,0 \mathrm{~cm}$ \\
4 & Proximal & $5,7 \mathrm{~cm}$ & $34,0 \mathrm{~cm}$ \\
& Distal & $8,0 \mathrm{~cm}$ & $30,5 \mathrm{~cm}$ \\
5 & Proximal & $9,0 \mathrm{~cm}$ & $26,5 \mathrm{~cm}$ \\
& Distal & $11,5 \mathrm{~cm}$ & $24,0 \mathrm{~cm}$ \\
6 & Proximal & $10,0 \mathrm{~cm}$ & $27,3 \mathrm{~cm}$ \\
& Distal & $14,0 \mathrm{~cm}$ & $23,3 \mathrm{~cm}$ \\
7 & Proximal & $7,5 \mathrm{~cm}$ & $31,0 \mathrm{~cm}$ \\
& Distal & $12,3 \mathrm{~cm}$ & $16,5 \mathrm{~cm}$ \\
8 & Proximal & $11,0 \mathrm{~cm}$ & $25,5 \mathrm{~cm}$ \\
& Distal & $8,5 \mathrm{~cm}$ & $23,0 \mathrm{~cm}$ \\
9 & Única & $16,7 \mathrm{~cm}$ & $28,0 \mathrm{~cm}$ \\
10 & Proximal & $9,5 \mathrm{~cm}$ & $36,0 \mathrm{~cm}$ \\
& Intermédia & $12,5 \mathrm{~cm}$ & $33,0 \mathrm{~cm}$ \\
& Distal & $16,2 \mathrm{~cm}$ & $29,3 \mathrm{~cm}$ \\
11 & Única & $8,5 \mathrm{~cm}$ & $35,0 \mathrm{~cm}$ \\
12 & Única & $7,5 \mathrm{~cm}$ & $36,0 \mathrm{~cm}$ \\
13 & Proximal & $10,5 \mathrm{~cm}$ & $25,5 \mathrm{~cm}$ \\
& Distal & $15,0 \mathrm{~cm}$ & $21,0 \mathrm{~cm}$ \\
\hline
\end{tabular}

\section{DISCUSSÃO}

Os retalhos miocutâneos de artérias perfurantes sedimentam-se na teoria dos angiossomos, proposta por Taylor et $a l^{(14-15)}$, em que uma determinada artéria perfurante cutânea é responsável pela nutrição de todas as estruturas correspondentes, desde o osso até a pele, como se fosse um bloco único, cujos limites são virtuais.

Dentre as vantagens dos retalhos de perfurantes encontram-se baixa morbidade ao sítio doador, preservação da irrigação axial, possibilidade de quimerismo (músculo e/ou pele e/ou periósteo, etc.) e dimensões variáveis dos retalhos. Têm-se como desvantagens a dificuldade de dissecção e a variabilidade no calibre e na localização das artérias perfurantes ${ }^{(16)}$.

Atualmente, dentre os retalhos de perfurantes mais empregados encontram-se os retalhos das artérias circunflexa lateral femoral (LCFAP), toracodorsal (TAP) e epigástrica inferior profunda (DIEP) ${ }^{(16)}$. Em 2001, Hallock $^{(10)}$ descreveu as bases anatômicas e o padrão vascular do MGM, proporcionando nova opção de retalho baseado em perfurantes.

Observou-se que a ASM divide-se em dois troncos intramusculares principais (um medial e outro lateral) e que as perfurantes originam-se do ramo lateral em $90 \%$ dos casos (Thione et al constataram 66\%). Em $38,5 \%$ dos casos, identificou-se uma perfurante e, em $46,1 \%$ dos casos, duas perfurantes (na casuística de Thione et al, 30 e $50 \%$, respectivamente). A quantidade de ramos perfurante variou entre nenhuma e três perfurantes, com média de 1,5 (em Thione et al, média de 1,9). Em uma peça anatômica, não se identificou nenhuma artéria perfurante, fato que não ocorreu nos estudos de Thione et $a l^{(12)}$ e Cavadas et $a l^{(11)}$, que observaram pelo menos uma perfurante.

No estudo radiográfico as artérias perfurantes emergiram a uma distância média da prega poplítea de $10,4 \mathrm{~cm}$, variando de 5,7 a $16,7 \mathrm{~cm}$, enquanto a distância ao maléolo medial variou de 16,5 a $36,0 \mathrm{~cm}$, com média de $28,4 \mathrm{~cm}$. Thione et al concluíram que todas as perfurantes encontravam-se entre 7,0 e $18,0 \mathrm{~cm}$ da prega poplítea, e que todas eram miocutâneas ${ }^{(12)}$.

\section{CONCLUSÃO}

A irrigação do MGM pela ASM e suas artérias perfurantes faz-se adequada para sua utilização como retalho miocutâneo no membro inferior.

\section{REFERÊNCIAS}

1. Dibbell DG. Edstrom LE. The gastrocnemius myocutaneous flap. Clin Plast Surg. 1980;7(1):45-50.

2. Aiache AE. A gastrocnemius muscle flap to fill an osteomyelitic hole in the femur. Br J Plast Surg. 1978;31(3):214-5.

3. Arnold PG, Mixter RC. Making the most of the gastrocnemius muscles. Plast Reconstr Surg. 1983;72(1):38-48.

4. Salibian AH, Rogers FR, Lamb RC. Microvascular gastrocnemius muscle transfer to the distal leg using saphenous vein grafts. Plast Reconstr Surg. 1984;73(2):302-7.

5. McCraw JB, Fishman JH, Sharzer LA. The versatile gastrocnemius myocutaneous flap. Plast Reconstr Surg. 1978; 62(1):15-23.

6. Ger R, Efron G. New operative approach in the treatment of chronic osteomyelitis of the tibial diaphysis. A preliminary report. Clin Orthop Relat Res. 1970;70:165-9.

7. Fujino T. Contribution of the axial and perforator vasculature to circulation in flaps. Plast Reconstr Surg. 1967;39(2):125-37. 
8. Koshima I, Soeda S, Yamasaki M, Kyou J. The free or pedicled anteromedial thigh flap. Ann Plast Surg. 1988;21(5):480-5.

9. Wei FC, Jain V, Suominen S, Chen HC. Confusion among perforator flaps: what is a true perforator flap? Plast Reconstr Surg. 2001;107(3):874-6. Comment in: Plast Reconstr Surg. 2002;109(4):1460-1; author reply 1461. Plast Reconstr Surg. 2004;114(5):1351.

10. Hallock GG. Anatomic basis of the gastrocnemius perforatorbased flap. Ann Plast Surg. 2001;47(5):517-22.

11. Cavadas PC, Sanz-Giménez-Rico JR, Gutierrez-de la Cámara A, Navarro-Monzonis A, Soler-Nomdedeu S, MartínezSoriano F. The medial sural artery perforator free flap. Plast Reconstr Surg. 2001;108(6):1609-15; discussion 1616-7.
12. Thione A, Valdatta L, Buoro M, Tuinder S, Mortarino C, Putz $\mathrm{R}$. The medial sural artery perforators: anatomic basis for a surgical plan. Ann Plast Surg. 2004;53(3):250-5.

13. Moraes FB, Paranahyba RM, Oliveira E, Kuwae MY, Rocha VL. Estudo anatômico do músculo gastrocnêmio medial visando transferência muscular livre funcional. Rev Bras Ortop. 2007; 42(8):261-5.

14. Taylor GI, Palmer JH. The vascular territories (angiosomes) of the body: experimental study and clinical applications. Br J Plast Surg. 1987;40(2):113-41.

15. Taylor GI. The angiosomes of the body and their supply to perforator flaps. Clin Plast Surg. 2003;30(3):331-42, v.

16. Geddes CR, Morris SF, Neligan PC. Perforator flaps: evolution, classification, and applications. Ann Plast Surg. 2003;50(1):90-9. 